\title{
Analysis and Realization of CAN Bus Technology in Industrial Conveyors
}

\author{
M. Nouby \\ South Valley University, \\ Qena, Egypt \\ Nouby.ghazaly@eng.svu.edu.eg
}

\begin{abstract}
Automation industry has undergone a drastic development in recent years. In this busy world human intervention in very high critical industrial processes are compensated by the enormous growth of the automation industries. Due to the advancement there is no need for a person to continuously monitor the system instead by implementing automation through latest technologies, a very high efficient operation of the system is possible. Conveyor system plays an important role in automation for material handlin $\mathrm{g}$ and packaging. For the movement of the material, that is transportation, in any processing, the lack of safety factor leads to many limitation in the automation, due to the unachievable efficiency, that is operational efficiency. For that variable speed drive are used for the purpose of modeling the conveyor belt speed, which is controlled through programmable logic controller. The main objective is to develop an embedded system that will automate the boiler control system by monitoring and controlling the temperature and pressure using the CAN bus communication technology. A PIC microcontroller is used in the project to control the entire operation of the embedded system..
\end{abstract}

Keywords-CAN bus, PIC, Boiler, Serial communication, Conveyors

\section{Introduction}

Throughout the years the interest for high caliber, more noteworthy productivity and computerized machines has expanded in the modern part of intensity plants. Force plants require ceaseless checking and review at visit stretches. There are potential outcomes of blunders at estimating and different stages engaged with human laborers and furthermore the absence of hardly any highlights of microcontrollers. The most significant part of any force plant is the evaporator control [1]. A few methods can be actualized to control the kettle in power plant. An evaporator is an encased vessel that gives a way to combustion warmth to be moved into water until it becomes warmed water or steam. The high temp water or steam under tension is then usable for moving the warmth for the steam necessities of procedure businesses or for power age. Verifiably, boilers were a wellspring of numerous genuine wounds and property obliteration because of ineffectively comprehended building standards. Over the top weight is possibly the most deadly type of heater mishap. These mishaps happen when the kettle cannot, at this point contain the exorbitant weight permitted to work in the heater. The administrator control, high weight limit, and the weight help valve need to flop before these mishaps can happen. Extreme weight mishaps, even in little boilers, have been known to totally decimate a structure [2].

In this way the traditional hardware frameworks are inclined to mistakes because of the association of people in the information assortment and handling utilizing entangled scientific articulations. In this way what we require is a framework that gathers crude information, forms it and presents it in values which can be confirmed and contrasted and the standard qualities. As of late, with the utilization of field-bus and implanted innovation in industry control territory the creation capacity is extraordinarily expanded. The expanding number of electronic gadgets, makes a sharp increment in level of trouble of wiring, and furthermore brings down the unwavering quality of the electrical framework. CAN-bus is a sequential correspondence organizes which adequately bolsters conveyed control and ongoing control, has been broadly utilized in the field of programmed control with its elite and high unwavering quality [3].

The purposes behind the predominant acknowledgment of CAN likewise incorporate utilization of a minimal effort, rapid configurable sequential, short information lengths offering low generally message, quick response times since bus ace or authorities are, inalienable unwavering quality because of complete blunder, capacity to communicate, multicast and uni-cast outlines and broad equipment execution of independent. CAN Bus stand apart for dependability, continuous and adaptability when contrasted and general correspondence bus since it embraces new advances and extraordinary plans. In this way CAN bus acquire the all inclusive applications in the modern control fields as one of most across the board field bus. By considering the goal to computerize the kettle control 
framework, the CAN bus innovation is actualized in controlling the basic parameters of evaporator like weight and temperature. The framework to be created will naturally control the parameters of the heater effectively [4].

Automation can be characterized as an innovation worried about the use of mechanical, electronic, and PC based framework to work and control creation [5]. The robotized components of the creation framework can be isolated into two classes: (1) Automation of the assembling frameworks in the plant, (2) Computerization of the assembling emotionally supportive network. In current creation framework, the two classes cover somewhat, on the grounds that the mechanized assembling frameworks working on the production line floor are themselves regularly actualized by PC framework and associated with the automated assembling emotionally supportive network and the board data framework working at the plant and undertaking level. The term PC incorporated assembling is utilized to show this broad utilization of PC underway framework [6].

Material dealing with is characterized as "the development, stockpiling, security and control of material all through the assembling and conveyance process including their utilization and removal". The treatment of material must be performed securely, effectively, effortlessly, in an ideal way, precisely, and without harm to the material. Material dealing with is a significant yet an ignored issue underway. The expense of material taking care of is a critical bit of all out creation cost. The extent shifts, contingent upon the sort of creation and level of automation in the material dealing with work. Plan of material dealing with framework must bring material trademark into contemplations [7], just as different variables like stream rate, directing element and booking. Programmable rationale controllers are entrenched for use in the execution of control calculation in numerous enterprises. First utilized in 1960's they were created to supplant hardwired electrical framework which had been already with the point of bringing the advantages of microchip control into the mechanical condition - explicitly to expand industrial facility control framework adaptability [8].

Customary mechanical automation guesses the administrator duty on observing and controlling of procedure progressively. As multifaceted nature of modern procedure builds, the requirement for remote controlling and checking from focal area increments too. This will make the administrator work simpler and capably [9][10].

\section{CAN ARCHITECTURE}

The Controller Area Network (CAN) is a sequential transport correspondences convention. It characterizes a standard for effective and solid correspondence between sensor, actuator, controller, and different hubs progressively applications. The control side microcontroller will set the control values for the temperature and pressure parameters. The actual temperature and pressure values from the master side controller are compared with the set values and the control action will be decided by the control side microcontroller. The block diagram of the embedded system to be developed to automate the boiler system is shown in figure 2. It consists of two PIC16F877A microcontrollers, in which one is referred as master side controller since the actions initiated for the control action is done in that particular controller and the other one is referred as control side controller as the control parameters and the exact control action to be implemented is decided in that particular controller. In this paper, temperature and pressure parameters are taken into consideration since these two parameters are the most critical parameters of a boiler. There is a temperature sensor module which measures the actual temperature of the boiler and that particular temperature value is given to the master side controller displayed in the LCD of the same controller. Similarly the pressure sensor module measures the actual pressure value and that particular pressure value is given to the master side controller which will be displayed in the LCD of the same controller. The information of the pressure and temperature values are transmitted over the CAN bus, through the CAN controller.

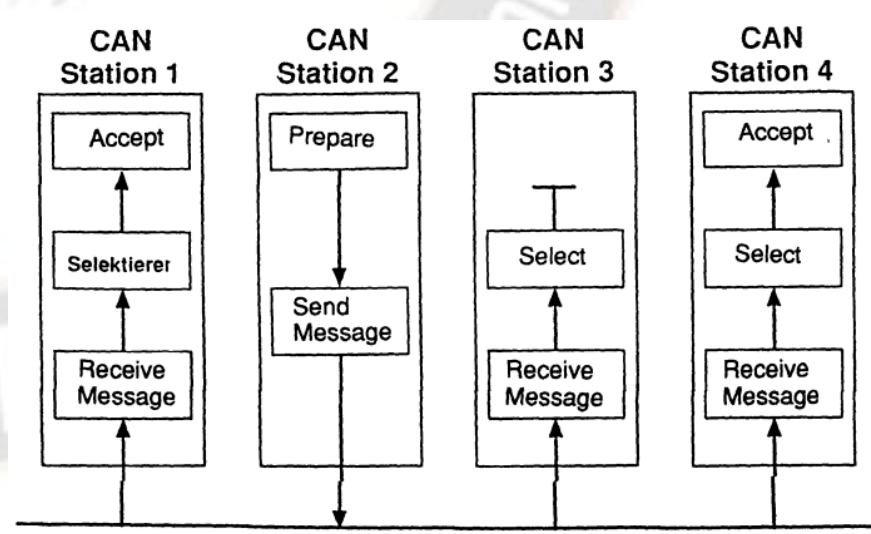

Fig 1: Structure of the CAN bus

If the value of the temperature exceeds the specified value, the voltage to the boiler is decreased therefore the temperature is brought to the specified value. If the temperature is less than the specified value the voltage to the boiler is increased, therefore the temperature is brought to the specified value. If the pressure is higher than the specified 
value, the valve is opened; therefore the pressure is brought to the specified value. If the pressure is less than the specified value the valve is closed therefore the pressure is brought to the specified value.In the material handling process safety is an important criterion. In this paper, conveyor system is implemented using GE Fanuc PLC, Variable Frequency Drive is used for controlling the speed of the conveyor during the material handling process. For the safety purpose, three layer SCADA architecture is used for supervisory and control. MODBUS protocol is used for interfacing, thus reducing the error, space and increasing the reliability respectively.

During the production or manufacturing process, the material should be transported at various speeds depending on the application. For low speed application, such as bottling units, if they are transported at high speed means, it leads to lot of problem. It even causes loss of accuracy to the end product and also loss of time in reconfiguring to those application and plant. The interfacing between the control and processing system have been done using analogue interface, which have made use of lot of wires and it had resulted in lot of failures.

\section{Proposed Methodology}

The Controller Area Network (CAN) is a successive exchanges show which profitably reinforces passed on nonstop control with a raised degree of security. Its space of utilization ranges from quick frameworks to insignificant exertion multiplex wiring. Controller Area Network (CAN) was from the start made by German vehicle structure supplier Robert Bosch in the mid-1980s for vehicle applications as a technique for enabling lively consecutive correspondence. The goal was to make cars continuously strong, safe and ecoaccommodating while at the same time reducing wiring saddle weight and multifaceted nature. Since its initiation, the CAN show has expanded wide commonness in mechanical computerization and vehicle/truck applications.

When data are transmitted by CAN, no stations are tended to, anyway rather, the substance of the message (for instance rpm or engine temperature) is doled out by an identifier that is exceptional all through the framework. The identifier describes the substance just as the need of the message. This is noteworthy for transport apportioning when a couple of stations are following vehicle get to. Figure. 2 shows the convey transmission by CAN centers. If the CPU of a given station wishes to establish a connection with at any rate one stations, it passes the data to be transmitted and their identifiers to the delegated CAN chip ("Make arranged"). This is all the CPU needs to do to begin data exchange. The message is fabricated and transmitted by the CAN chip. At the point when the CAN chip gets the vehicle task ("Send Message") each and every other station on the CAN compose become ON.

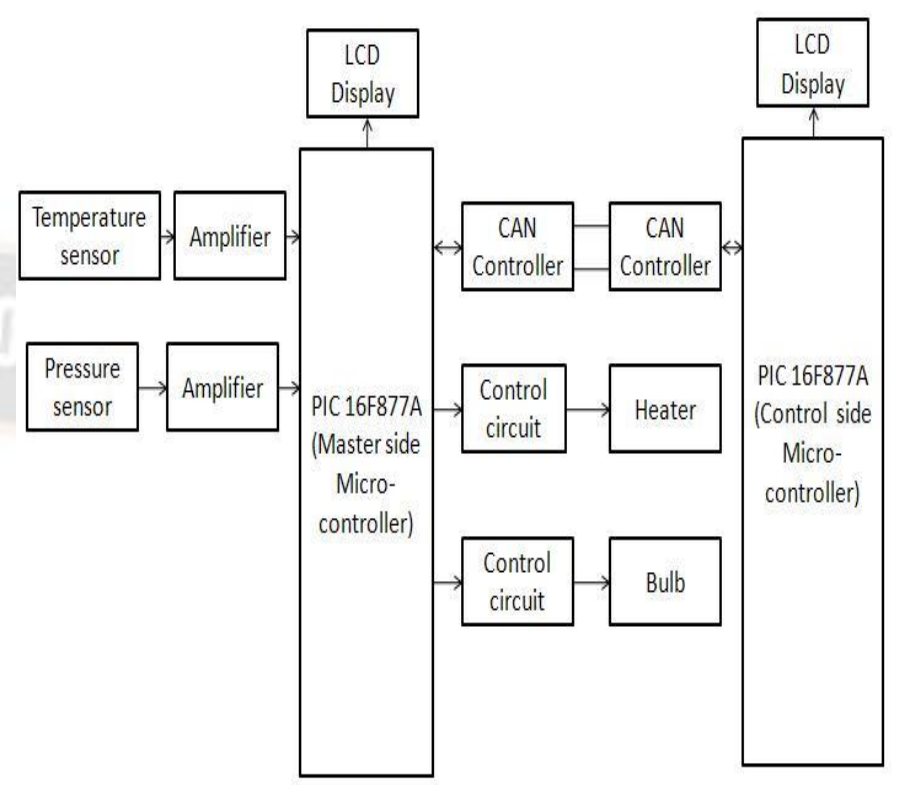

Fig 2: CAN Bus Technology in Industrial Conveyors

The information is of essentialness for the station concerned they are prepared ("Accept"), else they are disregarded. A high level of framework and setup adaptability is accomplished because of the con tent-situated tending to plot. It is extremely simple to add stations to the current CAN organize without making any equipment or programming alterations to the current stations, given that the new stations are absolutely recipients. Since the information transmission convention doesn't require physical goal addresses for the individual segments, it bolsters the idea of measured gadgets and furthermore allows various gathering (communicate, multicast) and the synchronization of circulated forms: estimations required as data by a few controllers can be transmitted through the system, so that it is superfluous for every controller to have its own sensor.

The conveyor system implemented in this paper is used for material handling. The logic control is done using GE-Fanuc Programmable Logic Controller. The supervisory and control is done using three-layer SCADA architecture. Thus, the reliability and safety of the materials are increased. The speed of the conveyor system is controlled using Variable Frequency Drive. Thus the operational efficiency of the conveyor system is increased. The control logic is programmed using ladder diagram (LD). Thus it enables to be reconfigured according to any application. The interface between supervisory control and process control is done 
using Mod-bus protocol. Thus errors due to analog wirings are reduced.

\section{Simulation Results}

The figure. 3 describes the flow of connection details between CAN-SPI module and PIC microcontroller. The temperature sensor and the pressure sensor modules are connected to any of the $\mathrm{A} / \mathrm{D}$ pin of the master side PIC microcontroller as the values from these devices will be analog values. These two values will be sent to other side of the PIC microcontroller through the CAN module. A keyboard and LCD display unit is connected to the control side microcontroller. This unit will be used to set the required temperature and pressure values. The comparison of the two parameters will be done on the control side microcontroller.

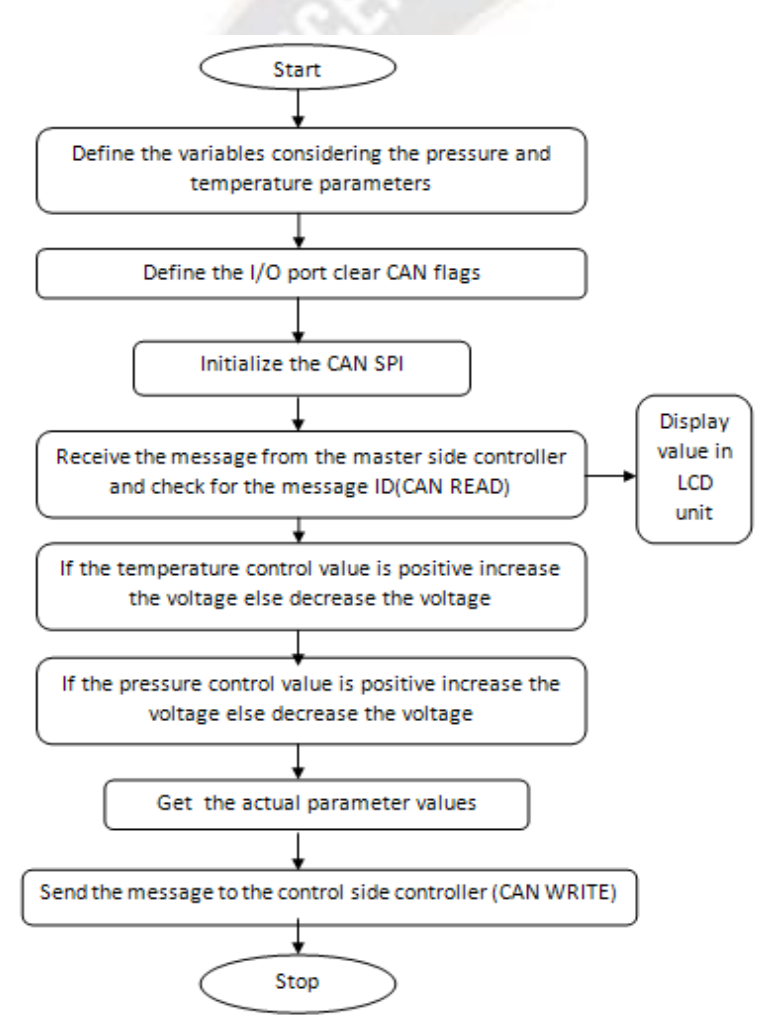

Fig 3: Flowchart showing the proposed system operation

Based on the required operation for the application is set in the Programmable Logic Controller. The input is set in PLC using the input key on the controller kit. The controller used here is GE Fanuc PLC. The control logic is programmed using Ladder Diagram (LD). Once the inputs are given and start button is pressed the conveyor starts to operate the transportation of the material. The required amount of speed that the conveyor rotates want to be set using PLC. Thus, on the defined input the Variable Frequency Drive operates the conveyor.

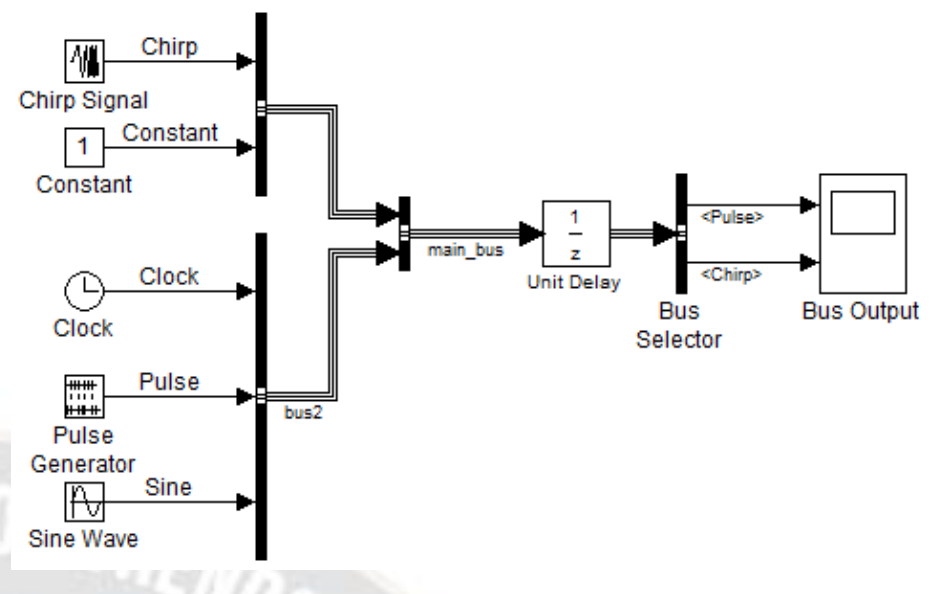

Fig 4: Simulink model of CAN bus

Multi-conveyors are used in this system. To transport the materials from bottom conveyor to top conveyor pneumatic arrangements i.e. pneumatic systems are used. SCADA is used for supervisory control of the system. Through SCADA the speed of the conveyor is controlled by interfacing the SCADA system with control, GE Fanuc, system. For safety purpose also SCADA is used for full shut-down of the system in order to prevent any damage to the material and parts of the system. The simulation results showing CAN bus realization in conveyor automation is shown in figure 5 .

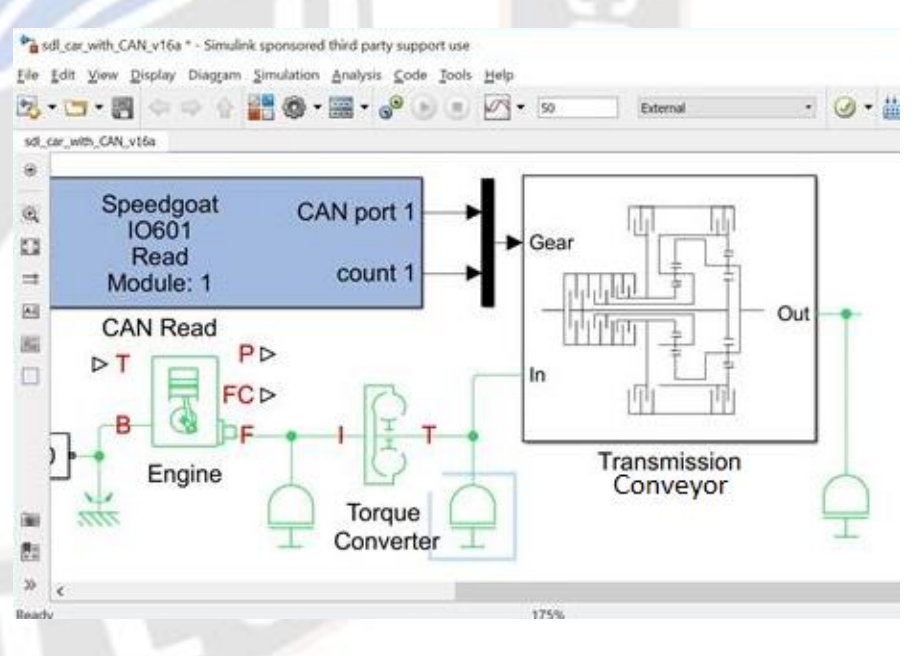

Fig 5: Simulink model of CAN bus interface with conveyor

The mechanical part of the multi-conveyor system is shown below. It consists of conveyor belts, pneumatic system, and Mild Steel (MS) stand for support and guide rollers. The VFD and motor are kept below the bottom conveyor. The CAN bus model values are tabulated in table 1 . 
International Journal on Recent and Innovation Trends in Computing and Communication

ISSN: 2321-8169 Volume: 8 Issue: 5

DOI: https://doi.org/10.17762/ijritcc.v8i5.5424

Article Received: 08 March 2020 Revised: 14 April 2020 Accepted: 07 May 2020 Publication: 31 May 2020

\begin{tabular}{|l|l|l|l|}
\hline \hline Data ID & $\begin{array}{l}\text { Data } \\
\text { (bytes) }\end{array}$ & Ti (ms) & Di (ms) \\
\hline SELECT $=1$ & 1 & 5 & 0.6 \\
\hline SELECT $=2$ & 2 & 4 & 0.4 \\
\hline SELECT $=3$ & 1 & 8 & 0.5 \\
\hline SELECT $=4$ & 2 & 4 & 0.7 \\
\hline SELECT $=5$ & 1 & 5 & 0.5 \\
\hline SELECT $=6$ & 2 & 6 & 0.4 \\
\hline SELECT $=7$ & 2 & 5 & 0.3 \\
\hline SELECT $=8$ & 6 & 4 & 0.2 \\
\hline SELECT $=9$ & 1 & 3 & 0.1 \\
\hline
\end{tabular}

Computer Systems and Applications, Tunis, pp.6-11, 2003.

[6] [10] Diaz, J. Rodriguez, E. Hurtado, L. Cacique, H. Vazquez, N. Ramirez, "CAN bus embedded system for lighting network applications circuits and systems", MWSCAS 2008. 51st Midwest Symposium on 10-13 Aug.2008, pp:531 - 534.

[7] Saju A. Kuruvilla, Swapna S. Gokhale, and Shivakumar Sastry (2008), 'Reliability Evaluation of Reconfigurable Conveyor Systems', 4th IEEE Conference on Automation Science and Engineering, pp.929-934.

[8] Salminen V and Verho A (1992), 'Multidisciplinary problems in mechatronics and some solutions', Computers in Electrical Engineering, vol. 18, no. 1, pp. 19.

\section{Conclusion}

The paper develops a prototype model which uses the CAN bus technology which can be implemented in any industrial processes where pressure and temperature are considered as main parameters. The CAN bus enables efficient communication between the controller modules. The prototype model can be extended and modified depending upon the application where it will be applied. The conveyor system presented in this paper is easily reconfigurable depending on the material to be handled. The control logic is implemented using GE Fanuc PLC using PME software. Thus, this can be reconfigured for any level of complication in the project. The main concept of controlling the speed is done using Variable Frequency Drive. This results in safety handling of the material in any application and improving the operational efficiency of the system. The supervisory control using three-layer architecture also helps in improving the safety measures and controlling the process from one place. The interface between the logic and supervisory control is done using CAN bus.

\section{References}

[1] Chu Liu, Feng Luo,Feng Luo, "A design for automotive CAN bus monitoring system," Vehicle Power and Propulsion Conference,2008(VPPC'08) .Sept,2008 pp:15.

[2] Cena,Gianluca, Valenzano, Adriano, "Improved CAN field bus for industrial applications," IEEE Transaction on Industrial Electronics, 1997,44(4):553-564.

[3] Farsi, M.; Ratcliff, K.; Barbosa, M.,'An overview of controller area network" Computing \& Control Engineering Journal,Vol 10, Issue 3, June 1999 Page(s): 113 - 120.

[4] Wargui, M.; Rachid, A. "Application of controller area network to mobile robots",Electrotechnical Conference, 1996. MELECON '96., 8th Mediterranean Volume 1, 1316 May 1996 Page(s):205 - 207 vol.1

[5] Syed Misbahuddin, Nizar Al-Holou, 'Efficient Data Communication Techniques for Controller Area Network (CAN) Protocol', ACS/IEEE International Conference on
[9] L. Tianming, "CAN bus application layer protocol design for space manipulator communication system," 2017 IEEE 2nd Advanced Information Technology, Electronic and Automation Control Conference (IAEAC), Chongqing, 2017, pp. 1627-1630, doi: 10.1109/IAEAC.2017.8054289.

[10] H. Wu, C. Chang and L. Li, "Research on CAN bus measuring technique based on collecting physical waveforms," 2017 IEEE 3rd Information Technology and Mechatronics Engineering Conference (ITOEC), Chongqing, 2017, pp. 887-890, doi: 10.1109/ITOEC.2017.8122481. 